La Revue

des Droits

de l'Homme

\section{La Revue des droits de l'homme}

Revue du Centre de recherches et d'études sur les droits fondamentaux

Actualités Droits-Libertés | 2019

\title{
Extension du domaine de la détention
}

À propos de l'arrêt de la Cour européenne des droits de l'homme, S., V. et A. c. Danemark, du 22 octobre 2018

\section{Vincent Sizaire}

\section{OpenEdition}

Journals

\section{Electronic version}

URL: http://journals.openedition.org/revdh/6179

DOI: 10.4000/revdh.6179

ISSN: 2264-119X

Publisher

Centre de recherches et d'études sur les droits fondamentaux

\section{Electronic reference}

Vincent Sizaire, "Extension du domaine de la détention », La Revue des droits de l'homme [Online], Actualités Droits-Libertés, Online since 14 February 2019, connection on 19 April 2019. URL : http:// journals.openedition.org/revdh/6179; DOI : 10.4000/revdh.6179

This text was automatically generated on 19 April 2019

Tous droits réservés 


\section{Extension du domaine de la détention}

À propos de l'arrêt de la Cour européenne des droits de l'homme, S., V. et A. c. Danemark, du 22 octobre 2018

\section{Vincent Sizaire}

1 Le supporter, nouvelle muse du législateur répressif ? Sans doute s'en seraient-ils passés mais, juste après la figure du terroriste, les aficionados des stades sont devenus en quelques années l'un des épouvantails les plus couramment brandis par les pouvoirs publics pour justifier l'adoption de mesures coercitives préventives supposées empêcher la commission d'actes de violence ${ }^{1}$. C'est ainsi que la Cour européenne des droits de l'homme a, dans un arrêt rendu en grande chambre le 22 octobre 2018, été amenée à se prononcer sur la conventionalité du dispositif danois de rétention policière préventif mis en œuvre à l'égard de supporters et, plus précisément, de la loi dite sur la police, qui lui permet de détenir une personne causant un « danger pour la sécurité des personnes et la sûreté publique $»^{2}$. Il s'agit cependant d'un mouvement qui traverse toute l'Europe, faisant dire à la Cour que « compte-tenu des nombreux cas où des rencontres de football ou d'autres manifestations de masse, sportives ou non, ont donné lieu à des violences au cours des dernières décennies en Europe, [elle] estime que l'on peut dire que la plupart des États membres doivent faire face aux mêmes difficultés " ${ }^{3}$.

2 C'est pourquoi sa décision doit être examinée avec la plus grande attention. Non seulement, parce qu'elle a vocation à fixer le cadre et, partant, les limites apportées au développement de cette singulière répression para-pénale, mais aussi parce qu'elle nous permet, ce faisant, d'apprécier la force de résistance de la Cour européenne des droits de l'homme à la montée en puissance de la logique répressive autoritaire que l'on observe sur tout continent. De ce point de vue, on ne peut que regretter que, au lieu d'adopter une interprétation stricte et exigeante du droit à la liberté et la sûreté tel que garanti par l'article 5 de la Convention, elle préfère se montrer «soucieuse d'interpréter et d'appliquer la Convention d'une manière qui tienne dûment compte des difficultés constatées tout en assurant une protection effective des droits de l'homme $»^{4}$. Et, ainsi, valider en son principe la possibilité, pour empêcher la commission d'une infraction, de 
recourir à une privation de liberté en dehors de toute procédure pénale. En vérité, tout se passe comme si, dans le droit fil de l'évolution de sa jurisprudence récente ${ }^{5}$, la Cour avait fait le choix d'accompagner un mouvement d'extension indéfini du domaine de la répression en se bornant à veiller au respect de garanties minimales.

Certes, le lecteur de ses arrêts est habitué aux circonvolutions diplomatiques dont elle fait régulièrement preuve pour ménager la marge d'appréciation des États et, plus largement, la susceptibilité de leurs représentants. Ce qui est en revanche nouveau - et préoccupant - c'est la propension croissante de la Cour de Strasbourg de reprendre à son compte les motifs d'opportunité avancés par les gouvernements pour justifier des atteintes portées aux droits protégés par la Convention. Une approche qui se veut explicitement l'expression d'une " certaine souplesse ${ }^{6}$, mais qui a surtout pour conséquence d'affaiblir la portée du contrôle de conventionalité et en particulier du contrôle de la proportionnalité des atteintes portées aux libertés. Une souplesse qui, dans le présent arrêt, conduit en outre la Cour à tordre de façon manifeste la lettre comme l'esprit de l'article 5 de la Convention. Ainsi que le font remarquer avec justesse les juges maltais et polonais dans leur opinion dissidente, une telle approche a quelque chose de «sidérant. Il y a dans la culture juridique européenne un principe communément admis selon lequel les dispositions qui garantissent la liberté personnelle sont d'interprétation stricte et littérale $\aleph^{7}$, principe d'ailleurs rappelé par la Cour elle-même au frontispice de son raisonnement ${ }^{8}$. L'extension du domaine de la détention qu'autorise ainsi la juridiction européenne s'avère ainsi très largement contra legem (I).

Toutefois, cette extension pourrait, à certains égards, n'être qu'une victoire à la Pyrrhus pour le camp autoritaire. Car, dans le même temps où elle valide la possibilité de détenir préventivement une personne en dehors de tout cadre pénal, la Cour européenne des droits de l'homme subordonne cette faculté au respect d'exigences suffisamment élevées pour questionner la conventionalité de dispositifs similaires d'ores et déjà mis en œuvre, en particulier en France (II).

\section{I/ - Une extension contra legem}

$5 \mathrm{Au}$ principe de l'assouplissement juridique revendiqué par la Cour, il y a un assouplissement herméneutique des plus singuliers. Pour déclarer conforme à la Convention la possibilité de détenir un individu en dehors de toute procédure pénale aux seules fins de prévenir la commission d'actes de violence, le juge européen est conduit à démembrer le texte de l'article 5.1.c de la Convention, pour donner une autonomie artificielle à cette forme de détention para-pénale (A). Une interprétation on ne peut plus élastique, qui le pousse dans le même mouvement à valider la possibilité d'une détention provisoire extrajudiciaire (B).

\section{A/ - La validation d'une détention provisoire ${ }^{9}$ para-pénale}

Depuis l'origine, la Convention autorise certes les États à priver de liberté une personne en dehors d'un cadre pénal. Il s'agit de la « détention régulière pour insoumission à une ordonnance rendue, conformément à la loi, par un tribunal ou en vue de garantir l'exécution d'une obligation prescrite par la loi », de celle « d'un mineur, décidée pour son éducation surveillée ou de sa détention régulière, afin de le traduire devant l'autorité compétente», de celle "d'une personne susceptible de propager une maladie 
contagieuse, d'un aliéné, d'un alcoolique, d'un toxicomane ou d'un vagabond » et enfin de celle « d'une personne pour l'empêcher de pénétrer irrégulièrement dans le territoire, ou contre laquelle une procédure d'expulsion ou d'extradition est en cours $»^{10}$. Mais, en dehors de ces hypothèses limitatives, et notamment lorsque les pouvoirs publics veulent parer à un risque, réel ou supposé, de violence ou de trouble à l'ordre public, un individu ne peut être privé de sa liberté à titre préventif que «s'il a été arrêté et détenu en vue d'être conduit devant l'autorité judiciaire compétente, lorsqu'il y a des raisons plausibles de soupçonner qu'il a commis une infraction ou qu'il y a des motifs raisonnables de croire à la nécessité de l'empêcher de commettre une infraction ou de s'enfuir après l'accomplissement de celle-ci $»^{11}$.

7 Jusqu'à l'arrêt du 22 octobre 2018, cette disposition était interprétée de façon constante et fort logiquement - comme conditionnant la possibilité de placer une personne en détention provisoire à l'existence d'une procédure pénale, au cours de laquelle elle se trouve, sous le contrôle d'un juge indépendant, mise en cause au titre de la commission d'une infraction déterminée ${ }^{12}$. Pour revenir sur cette interprétation, la Cour va se livrer à une glose pour le moins alambiquée. Dans un premier temps, elle isole du texte le motif de détention tiré de la nécessité d'empêcher une personne de commettre une infraction, en affirmant dorénavant «qu'il doit être considéré comme posant un motif de privation

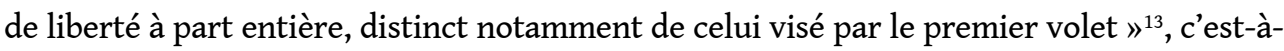
dire l'existence de raisons plausibles de soupçonner qu'elle a commis une infraction. Sur ce point, il est vrai que l'analyse purement grammaticale du texte, qui les évoque de façon alternative, peut permettre de conclure que les motifs de placement en détention provisoire autorisés par le Convention sont distincts. Dans un second temps, le juge européen croit pouvoir déduire de cette distinction que, "pour que les policiers ne se trouvent pas dans l'impossibilité pratique d'accomplir leur devoir de maintien de l'ordre et de protection du public, il faut en principe qu'ils puissent en vertu du paragraphe $1 \mathrm{c}$ ) de cet article procéder à des privations de liberté hors du cadre d'une procédure pénale » ${ }^{14}$. En d'autres termes, elle valide la possibilité pour les États de recours à une détention provisoire para-pénale, c'est-à-dire de priver une personne de sa liberté pour prévenir la commission d'une infraction mais sans pour autant s'inscrire dans une procédure tendant à sa constatation ou à sa sanction.

8 Et c'est sur ce point que l'on ne saurait suivre la Cour dans les méandres de ses assouplissements. Car, si l'on peut théoriquement admettre l'autonomie des motifs de privation de liberté énoncés par le paragraphe $1 \mathrm{c}$ ) de l'article 5 , il n'en demeure pas moins que, en toute hypothèse, c'est toujours « en vue d'être conduit devant l'autorité judiciaire compétente » que ladite privation est autorisée par la Convention ${ }^{15}$. Or on a du mal à imaginer, en pratique, que la présentation devant un juge, qui doit constituer la finalité pour laquelle la privation de liberté d'une personne suspectée de vouloir commettre une infraction a été ordonnée, puisse avoir d'autre objet que d'apprécier le bien-fondé de cette suspicion - et, ainsi, s'inscrire dans un autre cadre que celui d'une procédure pénale. Si la Cour était allée jusqu'au bout de son raisonnement tendant à valider le principe d'une détention para-pénale, elle aurait certes pu considérer que cette dernière n'était possible qu'à la condition que la personne n'ait été arrêtée qu'en vue d'être présentée devant une autorité judiciaire non pénale. Mais, outre, qu'on peine à imaginer la nature et l'office d'une telle juridiction, la démarche de la Cour vise en réalité à permettre à la seule police de "jouir d'une certaine marge d'appréciation dans l'adoption de décisions opérationnelles $»^{16}$. C'est dont fort logiquement que, s'éloignant 
encore davantage du texte de la Convention, elle en vient à valider le principe d'une détention provisoire extrajudiciaire.

\section{B/ - La validation d'une détention provisoire extrajudiciaire}

9 Rarement un arrêt de la Cour européenne des droits de l'homme aura été rédigé en usant de façon aussi manifeste du conditionnel. Ainsi, la Cour nous explique que «lorsqu'un individu est libéré après avoir fait l'objet d'une courte privation de liberté préventive, soit parce que le risque a disparu soit, par exemple, parce qu'un délai légal court a expiré, l'exigence selon laquelle les autorités doivent avoir pour but lorsqu'elles privent la personne de liberté de la conduire devant l'autorité judiciaire compétente ne devrait pas en elle-même faire obstacle à une privation de liberté préventive relevant du second volet de l'article 5 \$ $1 \mathrm{c}$ )». Et, effectivement, le conditionnel est de mise tant, quand bien même on considère qu'elle ne devrait pas le dire, la Convention énonce de façon on ne peut plus explicite que toute privation de liberté motivée par la nécessité d'empêcher la commission d'une infraction ne peut être décidée que parce que l'on envisage de déférer la personne devant l'autorité judiciaire. C'est une condition sine qua non de la conventionalité de la détention provisoire, quel que soit son motif.

10 Pour juger du contraire, la grande chambre fait d'abord valoir que l'article 5 de la Convention, ainsi qu'elle l'a précédemment jugé, n'est nullement méconnu dans l'hypothèse où la personne détenue est libérée avant sa présentation à l'autorité judiciaire et qu'elle n'est jamais, par la suite, poursuivie devant une juridiction pénale ${ }^{17}$. Ceci est indéniable mais n'enlève rien à l'exigence, posée par le texte, que cette privation de liberté ait été initialement ordonnée dans cette perspective. Toute différente est la situation qui lui était soumise et dans laquelle il est constant que les autorités n'ont jamais eu l'intention de présenter les personnes détenues à une autorité judiciaire, pour la bonne et simple raison que la procédure mise en œuvre n'a absolument pas cette finalité. La circonstance que la libération soit, en l'espèce, intervenue rapidement après l'arrestation, n'enlève rien au fait qu'elle n'a jamais eu pour objet d'assurer la comparution des individus privés de libertés devant un juge. Si la grande chambre tente également de justifier sa position en rappelant qu'elle admet depuis maintenant plus de vingt ans que les articles 2 et 3 la Convention imposent aux États de protéger le public contre les infractions ${ }^{18}$, une telle obligation ne saurait les exonérer du respect des autres droits qu'elle énonce, à commencer par le droit à la sûreté prévu à l'article 5. Elle ne saurait d'autant moins justifier le recours à une détention provisoire administrative que le droit pénal des États membres offre d'ores et déjà aux autorités de nombreux outils pour interpeller les personnes susceptibles de commettre, de façon imminente, des actes de violence - ne serait-ce qu'en les mettant en cause au titre de la tentative.

11 L'interprétation adoptée par la Cour dans le présent arrêt apparaît ainsi contraire non seulement à la lettre de la Convention, mais aussi à son esprit. Circonscrire à la procédure pénale la possibilité de placer une personne en détention provisoire constitue une importante garantie contre une privation de liberté arbitraire. Non seulement parce qu'elle suppose l'existence d'indices matériels laissant penser que la personne a commis un crime ou un délit, mais aussi parce qu'elle suppose l'intervention d'une autorité judiciaire qui, comme le prévoit par ailleurs l'article 6 de la Convention, doit présenter toutes garanties d'indépendance et d'impartialité. En revenant sur cette position, la Cour ouvre la porte au recours, par les États membres, à une détention provisoire ordonnée à 
titre purement préventif par le seul pouvoir exécutif, sans que la personne ne dispose d'autre recours juridictionnel qu'une hypothétique action en réparation d'une privation de liberté irrégulière ${ }^{19}$. Une approche qui n'apparaît guère de nature à renforcer le droit à la liberté et à la sûreté des citoyen-n-es européen-n-es. À cet égard, il est significatif de relever que, pour tenter d'assoir sa position, la Cour exhume un vieil arrêt par lequel ses prédécesseurs avaient, en 1961, validé le dispositif de rétention administrative des membres de «l'armée républicaine irlandaise » (IRA) par les autorités britanniques ${ }^{20}$. Un arrêt qui, comme le rappellent les auteurs de l'opinion dissidente "a été rendu à une époque où la pleine judiciarisation de la Convention dépendait des États qui l'avaient ratifiée et qui avaient accepté ce qui n'était jusque-là qu'un ensemble de dispositions facultatives $»^{21}$.

Toutefois, si elle se réfère à plusieurs reprises à cet arrêt, la Cour subordonne dans le même temps le recours à ce mode spécifique de détention au respect de différentes conditions qui rendraient aujourd'hui manifestement inconventionnel le dispositif répressif britannique. Des conditions qui, à l'analyse, apportent de sérieuses limites à l'extension de cette singulière coercition para-pénale.

\section{II/ - Une extension contenue}

13 L'interprétation pour le moins baroque de l'article 5 de la Convention à laquelle s'est livrée la Cour pour valider la privation de liberté para-pénale qui lui était soumise la conduit, paradoxalement, à enserrer cette nouvelle forme de détention dans un cadre juridique très strict, tant en ce qui concerne son domaine d'application (A), que ses conditions de validité (B).

\section{A/ - Une détention circonscrite}

La validation de la détention para-pénale au visa l'article 5 \$3 c) de la Convention n'a certes pu se faire qu'au prix d'acrobaties herméneutiques d'une rare souplesse. À défaut d'interprétation stricte, on peut néanmoins juger une telle solution préférable à celle que le gouvernement danois proposait à la Cour d'adopter : la validation de son dispositif de rétention policière préventive au visa de l'article $5 \S 3 \mathrm{~b}$ ), c'est-à-dire comme une « détention régulière [...] en vue de garantir l'exécution d'une obligation prescrite par la loi ». Une obligation se résumant, en l'espèce, à ne pas causer « de danger pour la sécurité des personnes et la sûreté publique »... Or, comme la Cour européenne des droits de l'homme le juge depuis plus de quarante ans, la méconnaissance d'une obligation prévue par la loi ne peut justifier une mesure privative de liberté que si elle présente un caractère suffisamment "spécifique et concret». À l'inverse, une "interprétation extensive entraînerait des résultats incompatibles avec l'idée de prééminence du droit dont s'inspire la Convention tout entière $»^{22}$. Pour le dire autrement, une telle interprétation ouvrirait la porte à des mesures privatives de liberté qui, fondées sur la méconnaissance d'obligations génériques et imprécises, seraient totalement arbitraires. Le risque existait pourtant que, en la matière également, la Cour ne procède à un revirement de jurisprudence : en 2013, elle avait jugé que le simple fait que la police donne un ordre précis à un supporter suspecté de vouloir commettre des violences suffisait à rendre suffisamment "spécifique et concrète" l'obligation de ne pas 
commettre d'infractions pénales et, ainsi, à justifier la privation de liberté qui lui avait infligée quand il y avait passé outre ${ }^{23}$.

Elle n'en a heureusement rien fait. En inscrivant la détention administrative dans un cadre para-pénal, la décision de la Cour a au moins le mérite d'en circonscrire rigoureusement le domaine d'application. Ainsi, une telle privation n'est jugée conforme à la Convention que si l'infraction dont il s'agit de prévenir la commission est « concrète et déterminée, notamment en ce qui concerne le lieu et le moment où elle serait commise ainsi que ses victimes potentielles [...], et que les autorités soient en mesure de produire des faits ou renseignements propres à persuader un observateur objectif que l'intéressé aurait selon toute probabilité participé à la commission de cette infraction concrète et déterminée s'il n'en avait pas été empêché par la privation de liberté dont il a fait l'objet » ${ }^{24}$. Sont prohibées de la sorte les mesures de détention qui ne seraient motivées que par un risque général de commission d'infractions pénales tout comme les mesures qui ne seraient prises qu'en considération du comportement de la personne.

Outre qu'elle condamne par avance toute velléité d'instituer une rétention administrative à l'égard des personnes supposément radicalisées sur la seule base de leur inscription sur un fichier - proposition régulièrement brandie dans le débat public français - une telle interprétation doit également nous conduire à questionner la conventionalité des nombreux dispositifs coercitifs de nature para-pénale adoptés en France ces dernières années. Ainsi les assignations à résidence prononcées à l'encontre des personnes revenant d'un "théâtre (sic.) d'opérations de groupements terroristes ${ }^{25}$ ou celles qui frappent les personnes dont le «comportement» est considéré comme "une menace d'une particulière gravité pour la sécurité et l'ordre publics $\aleph^{26}$, peuvent être ordonnées sans que les autorités n'aient à justifier de la nécessité de prévenir la commission d'une infraction déterminée. Certes, il ne s'agit pas, formellement, de mesures privatives de liberté et l'on sait que le Conseil constitutionnel a une acception particulièrement "souple» de la notion de restriction de liberté27. Cependant, la Cour européenne considère pour sa part qu'entre "privation et restriction de liberté, il n'y a [...] qu'une différence de degré ou d'intensité, non de nature ou d'essence ", de sorte qu'une mesure qualifiée de restriction de liberté peut, si elle conduit à soumettre la personne à un régime particulièrement coercitif, être assimilée à une privation de libertée ${ }^{28}$. Sous ce rapport, certaines des assignations à résidence administratives qui sont aujourd'hui prononcées pourraient dorénavant, en fonction de l'importance des sujétions qu'elles imposent aux personnes assignées, être jugées contraires à l'article 5 de la Convention européenne de sauvegarde des droits de l'homme et des libertés fondamentales. De la même façon, les autres conditions auxquelles la Cour subordonne la validité de la détention para-pénale peuvent nous conduire à (re)mettre en cause la conventionalité de certaines pratiques répressives nationales.

\section{B/ - Une détention encadrée}

Problématique en son principe, la validation par la Cour européenne d'une détention provisoire en dehors de toute procédure pénale n'en demeure pas moins « limitée par les garanties importantes posées à l'article $5 \S 1 »^{29}$. Dans une déclaration de principe solennelle et didactique qui peut se lire comme une forme de contrepartie à la "souplesse » dont elle se prévaut par ailleurs, la Cour rappelle ainsi que cette privation de liberté n'est admissible que si elle respecte le cadre légal qui l'a instituée, qu'elle est 
strictement nécessaire - ce qui suppose que les autorités démontrent que d'autres mesures, moins sévères, ont été considérées et jugées insuffisantes - et qu'elle est dénuée d'arbitraire. En particulier, la Cour rappelle qu'une détention est «arbitraire lorsque, même si elle est parfaitement conforme à la législation nationale, il y a eu un élément de mauvaise foi ou de tromperie de la part des autorités $»^{30}$. Un rappel qui peut sonner comme un truisme mais qui apparaît en réalité tristement nécessaire à une époque où la plasticité des critères permettant la mise en œuvre des mesures coercitives para-pénales permet leur extension bien au-delà des motifs officiels ayant présidé à leur institution. Ainsi, il est frappant de constater que les perquisitions et assignations à résidence administratives ordonnées entre novembre 2015 et octobre 2017 sous couvert d'un état d'urgence proclamé pour lutter contre la criminalité terroriste ont été, très rapidement, utilisées à l'encontre de militants présentés comme appartenant à une supposée « mouvance contestataire radicale ${ }^{31}$.

Par ailleurs, la Cour vient encadrer de façon rigoureuse la durée de cette détention provisoire para-pénale dont elle valide le principe, en estimant cette fois - au risque de souligner un peu plus les contradictions émaillant son raisonnement - que «la stricte limite de temps imposée par [la Convention] ne laisse guère de souplesse dans l'interprétation $»^{32}$. Considérant que «la durée nécessaire entre l'arrestation de la personne à titre préventif et sa comparution à bref délai devant un juge ou un autre magistrat doit être plus courte que dans le cas d'une détention provisoire en matière pénale ", elle en déduit que « de manière générale, dans le cas d'une privation de liberté préventive, une libération intervenant "plus tôt qu'un contrôle juridictionnel à bref délai » doit intervenir au bout de quelques heures et non au bout de quelques jours $»^{33}$. Une telle interprétation présente l'avantage de limiter strictement le recours à ce type de mesure à un moment où elles sont en voie de généralisation en Europe.

De façon plus inattendue, elle apparait en outre de nature à remettre en cause des pratiques répressives qui, à la faveur du durcissement de la réaction des pouvoirs publics face à certains mouvements sociaux, reviennent aujourd'hui sur le devant de la scène. Ainsi, en particulier, des gardes à vue ordonnées aux seules fins d'empêcher la circulation de manifestants et dont on a vu le nombre exploser à la faveur de la révolte dite des gilets jaunes ${ }^{34}$. Des mesures décidées formellement dans un cadre pénal mais qui n'ont en réalité qu'une fonction purement préventive. La plupart de celles qui ont été prises à l'encontre des manifestant-e-s en gilet l'ont d'ailleurs été sur le fondement du délit de participation à un groupement violent, qui fut très officiellement introduit pour permettre à la police d'arrêter préventivement - et au risque d'un grand arbitraire - des individus supposément violents ${ }^{35}$. À partir du moment où il est établi que, dès le début de la garde à vue, les autorités n'avaient aucune intention de poursuivre la personne en justice ne faudrait-il pas considérer désormais que nous nous trouvons dans le cadre de ce nouveau motif de détention para-pénale, ce qui suppose une privation de liberté limitée à "quelques heures»? Certes, cela serait faire preuve d'une certaine souplesse... Mais n'est-ce pas le nouvel état d'esprit auquel nous invite la Cour européenne des droits de l'homme?

Cour EDH, GC, S., V. et A. c. Danemark, 22/10/2018, Req. $\mathrm{n}^{\text {os }} 35553 / 12,36678 / 12$ et 36711/12 
Les Lettres « Actualités Droits-Libertés » (ADL) du CREDOF (pour s'y abonner) sont accessibles sur le site de la Revue des Droits de l'Homme (RevDH) - Contact

\section{NOTES}

1. Vincent Sizaire, "Du stade au Laboratoire, surveiller et punir les supporters », in Délibérée n 6 6, février 2019.

2. Article 5 de la loi no 444 du 9 septembre 2004.

3. CEDH, S., V. et A., c/ Danemark, cons 94.

4. Ibid., cons 95.

5. V ; par exemple, CEDH, Centrum för Rättvisa c. Suède, 19 juin 2018, requête $n^{\circ} 35252 / 08$, ou CEDH 13 septembre 2018, Big Brother Watch et autres c. Royaume-Uni, $n^{\circ}$ s58170/13, 62322/14 et 24960/15), ou la Cour valide en leur principe les dispositifs d'interception massive des données de communication mis en place par les services de renseignement.

6. CEDH, S., V. et A., c/ Danemark, cons 118.

7. Opinion dissidente des juges de Gaetano et Wojtyczek, cons.5.

8. CEDH, S., V. et A., c/ Danemark, cons 73.

9. Il est ici fait référence à la notion telle qu'elle est utilisée par la Cour européenne des droits de l'homme, et qui englobe tant la détention provisoire stricto sensu que la garde à vue.

10. Article $5 \S 1 \mathrm{~b}$ ), d), e) et f) de la Convention européenne de sauvegarde des droits de l'homme et des libertés fondamentales.

11. Article $5 \$ 1 \mathrm{c}$ ) de la Convention européenne de sauvegarde des droits de l'homme et des libertés fondamentales.

12. CEDH, Ciulla c. Italie, 22 février $1989, \mathrm{n}^{\circ} 11152 / 84$.

13. CEDH, S., V. et A., c/ Danemark, cons 114.

14. Ibid, cons 116 .

15. Article $5 \$ 1 \mathrm{c}$ ) de la Convention européenne de sauvegarde des droits de l'homme et des libertés fondamentales.

16. CEDH, S., V. et A., c/ Danemark précité, cons 123.

17. CEDH AFFAIRE BROGAN ET AUTRES c. Royaume-Uni, 29 novembre 1988, (Requête no $11209 / 84 ; 11234 / 84 ; 11266 / 84 ; 11386 / 85)$.

18. Cons 124.

19. Article $5 \$ 5$ de la Convention européenne de sauvegarde des droits de l'homme et des libertés fondamentales.

20. CEDH, Lawless c/Irlande, $1^{\text {er }}$ juillet 1961.

21. Opinion dissidente des juges de Gaetano et Wojtyczek, cons.4.

22. CEDH, ENGEL ET AUTRES c. PAYS-BAS, 8 juin 1976 (Requête no 5100/71; 5101/71; 5102/71 ; $5354 / 72 ; 5370 / 72)$ cons 69.

23. CEDH, OSTENDORF v. GERMANY, 15598/08 07/03/2013

24. CEDH, S., V. et A., c/ Danemark précité, cons 127. 
25. Article L.225-1 du code de la sécurité intérieure, introduit par la loi 2016-731 du 3 juin 2016.

26. Article L.229-1 du code de la sécurité intérieure, introduit par la loi 2017-1510 du 30 octobre 2017.

27. Il estime ainsi qu'une assignation à résidence d'une personne en dehors de son domicile ne constitue pas une mesure privative de liberté pour peu qu'elle n'excède pas douze heures ; CC, Décision $n^{\circ}$ 2015-527 QPC du 22 décembre 2015, considérant $n^{\circ} 6$.

28. CEDH, GUZZARDI c. Italie, 06/11/1980, $n^{\circ} 7367$, cons 93.

29. CEDH, S., V. et A., c/ Danemark précité, cons 127.

30. Ibid., cons. 76.

31. S. Hennette Vauchez, M. Kalogirou, N. Klausser, C. Roulhac, S. Slama et V. Souty, "l'état d'urgence au prisme du contentieux : analyse transversale du corpus », in S. Hennette Vauchez (dir.), Ce qu'il reste(ra) toujours de l'état d'urgence, LGDJ, Paris, 2018, p. 270 et suivantes.

32. CEDH, S., V. et A., c/ Danemark précité, cons 130.

33. Ibid., cons 133 et 134.

34. V. Raphaël Kempf, "Des violences policières aux violences judiciaires ", Le monde diplomatique, février 2019.

35. Article 222-14-2 du Code pénal, institué par la loi $n^{\circ} 2010-201$ du 6 mars 2010, issu d'une proposition enregistrée à l'Assemblée nationale sous le n 1641 le 5 mai 2009 dont l'auteur indiquait « cet article répond pleinement à un objectif de prévention. Il permettra de sanctionner les membres qui, en connaissance de cause, appartiennent à un groupement ayant des visées violentes, avant même que cette bande ne commette un délit déjà prévu par le Code pénal ».

\section{ABSTRACTS}

$\mathrm{Au}$ bénéfice d'une souplesse revendiquée et, en vérité, d'une lecture très largement contra legem de l'article 5 de la Convention européenne des droits de l'Homme, la Cour de Strasbourg a, dans son arrêt de grande chambre du 28 octobre 2018 S., V. et A., c/ Danemark, étendu le domaine de la détention en validant le principe d'une singulière détention para-pénale. Toutefois, en assortissant cette reconnaissance de conditions très strictes, cet arrêt constitue aussi, paradoxalement, un frein au développement de la logique répressive administrative qui se déploie en Europe depuis près de vingt ans.

\section{AUTHOR}

\section{VINCENT SIZAIRE}

Maître de conférences associé à l'Université de Paris Nanterre 